\title{
Target-Related and Intrinsic Neuronal Death in Lurcher Mutant Mice Are Both Mediated by Caspase-3 Activation
}

\author{
Fekrije Selimi, Martin Doughty, Nicole Delhaye-Bouchaud, and Jean Mariani \\ Laboratoire Développement et Vieillissement du Système Nerveux, Institut des Neurosciences, Unité Mixte de Recherche \\ 7624, Centre National de la Recherche Scientifique et Université Pierre et Marie Curie, 75005 Paris, France
}

The Lurcher $(L c)$ mutation in the $\delta 2$ glutamate receptor gene leads to the presence of a constitutive inward current in the cerebellar Purkinje cells of Lurcher heterozygous mice and to the postnatal degeneration of these neurons. In addition, cerebellar granule cells and olivary neurons of $L c /+$ mice die as an indirect effect of the mutation after the loss of their target Purkinje cells. The apoptotic nature of $L c /+$ Purkinje cell death remains controversial. To address this question, we studied the involvement of caspase-3, a key effector of apoptosis, in the neurodegenerative processes occurring in $\mathrm{LC} /+$ cerebellum. Several antibodies recognizing different regions of caspase-3 were used in immunoblotting and immunohistochemical experiments. We demonstrate that pro-caspase-3 is specifically up- regulated in the dying Lc/ + Purkinje cells, but not in granule cells and olivary neurons, suggesting that different deathinducing signals trigger variant apoptotic pathways in the CNS. The subcellular localization of pro-caspase-3 was shown to be cytoplasmic and mitochondrial. Active caspase-3 as well as DNA fragmentation was found in numerous granule cells and some Purkinje cells of the $L c /+$ cerebellum. Thus, caspase- 3 activation is involved in both the direct and indirect neuronal death induced by the Lurcher mutation, strongly supporting the idea that the $L c /+$ Purkinje cell dies by apoptosis.

Key words: caspase-3; neuronal death; Lurcher; Purkinje cells; apoptosis; TUNEL; immunohistochemistry
The neurological mutant mice Lurcher $(L c /+)$ are characterized by a severe ataxia caused by a defect of the cerebellum. The cerebellum of $L c /+$ mice is affected by a progressive postnatal degeneration of $>90 \%$ of the Purkinje cells and by large-scale death of granule cells and olivary neurons after the loss of target Purkinje cells (Caddy and Biscoe, 1979). The semi-dominant Lurcher mutation $(L c)$ is lethal when homozygous and has been identified as a point mutation in the $\delta 2$ glutamate receptor gene (GluR $\delta 2$ ) located on chromosome 6. The GluR $\delta 2$ protein is expressed specifically in cerebellar Purkinje cells (Araki et al., 1993) and has an unspecified function. The G-to-A transition found in the $L c$ allele is a gain-of-function mutation, resulting in a large, constitutive inward current in $L c /+$ Purkinje cells, suggesting that the cell dies by excitotoxicity (Zuo et al., 1997). It has been proposed that a $L c /+$ Purkinje cell dies by an apoptotic mechanism (Norman et al., 1995; Wüllner et al., 1995, 1998). However, previous morphological studies have concluded that $L c /+$ Purkinje cell death is necrotic (Dumesnil-Bousez and Sotelo, 1992). Thus, the precise nature of the mechanism underlying the $L c /+$ Purkinje cell death remains unclear and needs to be resolved by the analysis of other characteristic features of apoptosis and necrosis. These types of cell death were originally distinguished by morphological features. Apoptosis is now also

\footnotetext{
Received May 3, 1999; revised Nov. 2, 1999; accepted Nov. 11, 1999.

This work was supported by European Community Biotech Grant BIO4C96 0774 and by grants from Fondation de la Recherche Médicale. Drs. Brown and Goldstein kindly provided us with the anti-hamster caspase-3 antibody, Dr. Nicholson with R280 and MF397 antibodies, and Dr. Srinivasan with CM1 antibody. We also thank P. Bouquet and the Centre Interuniversitaire de Microscopie Electronique for technical assistance, M. Vesleau for photographic assistance, and C. Chianale for breeding of the mutants.

Correspondence should be addressed to Fekrije Selimi, Institut des Neurosciences, Université Pierre et Marie Curie, Boite 14, Bâtiment B, 4 ème étage, 9 Quai Saint Bernard 75005 Paris, France. E-mail: Fekrije.Selimi@snv.jussieu.fr. Copyright (C) 2000 Society for Neuroscience 0270-6474/00/200992-09\$15.00/0
}

characterized by the involvement of caspases. Caspases are structurally similar aspartate-specific cysteine proteases. They are synthesized as a precursor, which is activated by cleavage. Three categories of caspases can be distinguished by the specificity of their substrate cleavage site (Nicholson and Thornberry, 1997; Thornberry et al., 1997): caspases generating mature proinflammatory cytokines (caspase-1, 4, 5), effector caspases (caspase-2, 3, 7 and CED3), and initiator caspases (caspase-6, 8, 9) of apoptosis. More specifically, caspase-3 activation has been demonstrated in several models of neurodegeneration in vitro ( $\mathrm{Du}$ et al., 1997; Marks et al., 1998). Caspase-3 is also necessary for developmental neuronal death because caspase- 3 knock-out mice have prominent protrusions of the brain tissue caused by the presence of supernumerary neurons in many regions of their brain (Kuida et al., 1996). However, the role of caspase-3 during pathological neuronal death in vivo remains largely unknown. Recently, Namura et al. (1998) demonstrated the expression and activation of caspase- 3 in apoptotic neurons after experimental cerebral ischemia, but the possible role of caspase- 3 in a pathological neuronal death of genetic origin has not been examined.

We have studied the expression and localization of caspase- 3 in the cerebellum of $L c /+$ mice. We show that caspase-3 is upregulated in degenerating $L c /+$ Purkinje cells, but not in $L c /+$ granule cells. In contrast, active caspase- 3 can be found in degenerating Purkinje cells, as well as in dying granule cells and olivary neurons. These data strongly favor the idea that $L c /+$ Purkinje cell death is apoptotic and suggest the involvement of caspase- 3 in a pathological neuronal death of genetic origin and in targetrelated cell death.

\section{MATERIALS AND METHODS}

Animals. Lurcher heterozygotes $(L c /+)$ and their control wild-type $(+/+)$ littermates were obtained by breeding $L c /+$ males with B6CBA $+/+$ females. $L c /+$ mice were recognized by their ataxia, and their 
identification was confirmed by the presence of an atrophic cerebellum. All animal procedures were performed under the guidelines established by "le comité national d'éthique pour les sciences de la vie et de la santé".

Immunoblotting. Protein extracts were prepared from cortex, hippocampus, and cerebellum of postnatal day 20 (P20) animals using the following lysis buffer: $1.6 \mathrm{~mm}$ Tris- $\mathrm{HCl}, \mathrm{pH} 8.0 ; 1.5 \mathrm{~mm} \mathrm{NaCl} ; 1 \%$ glycerol; $0.005 \%$ sodium deoxycholate; $0.0025 \%$ igepal; and $0.0001 \%$ lauryl sulfate. The lysis buffer contained the following protease inhibitors: $1 \mathrm{~mm}$ 4-(2-aminoethyl)-benzenesulfonyl fluoride hydrochlorine; $0.5 \mathrm{~mm}$ iodoacetamide; $0.1 \mathrm{~mm}$ leupeptin; $0.02 \mathrm{mg} / \mathrm{ml}$ soybean trypsin inhibitor; $1 \mu \mathrm{M}$ pepstatin; and $2 \mu \mathrm{g} / \mathrm{ml}$ aprotinin. The protein concentration was measured in each extract using the Bio-Rad (Hercules, CA) DC protein assay based on the Lowry assay. Protein extraction was repeated at least three times. Ten micrograms of proteins were separated by SDS-PAGE using gels with a gradient of acrylamide (10-20\%; Bio$\mathrm{Rad})$ and transferred on a Hybond-C super nitrocellulose membrane (Amersham, Buckingham, UK). Membranes were saturated using 5\% milk (diluted in PBS containing $0.2 \%$ Tween 20 ) and incubated overnight at $4^{\circ} \mathrm{C}$ with an anti-hamster caspase- 3 antiserum (1:2000 dilution, a kind gift of Drs. Brown and Goldstein) in PBS, $0.2 \%$ Tween 20, and 5\% milk. This antiserum is directed against a peptide located in the N-terminal part of hamster caspase- 3 and recognizes both the precursor and the p20 subunit of caspase-3 (Wang et al., 1996). The membranes were washed, and the immunocomplex was detected using a peroxidase-conjugated anti-rabbit IgG (Jackson ImmunoResearch, West Grove, PA) and diaminobenzidine tetrahydrochloride (DAB) revelation (DAB Sigmafast; Sigma, St. Louis, MO). Similarly, $20 \mu \mathrm{g}$ of proteins of each extract were separated and transferred for immunoblotting with R280 antibody that recognizes only the precursor form of caspase-3 (a kind gift of Dr. Nicholson), using a protocol previously described (Rodriguez et al., 1996). Densitometry measurements were performed on three separate experiments using an image analysis system (DensyLab; Bioprobe Systems, Montreuil, France).

Immunohistochemistry. Animals at P12, P15, and P20 were anesthetized by an intraperitoneal injection of $3.5 \%$ chloralhydrate $(0.1 \mathrm{ml} / \mathrm{gm}$ weight) and perfused with $0.9 \% \mathrm{NaCl}$, followed by $95 \%$ ethanol. Brains were dissected, incubated overnight in Clarke's fixative, and processed for paraffin-embedding. Ten-micrometer-thick tissue sections (either parasagittal or coronal) were processed for double immunostaining using the anti-hamster caspase-3 antiserum and an anti-calbindin monoclonal antibody (CL-300; Sigma). Nonspecific binding sites were blocked using $4 \%$ normal horse serum and $4 \%$ normal goat serum diluted in TBS and $0.2 \%$ Triton $\mathrm{X}-100$. The sections were then incubated overnight with the anti-hamster caspase- 3 antibody (dilution $1: 150$ in TBS and $0.2 \%$ Triton X-100) and CL-300 antibody (1:200). Caspase-3 immunolabeling was detected using an FITC-conjugated anti-rabbit antibody (Jackson ImmunoResearch), and calbindin immunolabeling using a Cy3-conjugated anti-mouse antibody (Jackson ImmunoResearch). In each experiment, control sections were included in which the anti-caspase- 3 antibody was replaced by normal rabbit serum (dilution 1:150 in TBS and $0.2 \%$ Triton $\mathrm{X}-100)$. At least two or three $L c /+$ and control animals were analyzed at each age.

Immunohistochemistry was performed on brain sections of $L c /+$ and $+/+$ mice (P15 and P20) using a rabbit polyclonal antibody directed against a peptide contained in the $\mathrm{C}$-terminal part of the p20 subunit of caspase-3 (CM1 antibody; a kind gift of Dr. Srinivasan). This antibody has been demonstrated to recognize the p20 subunit of active caspase-3 (Namura et al., 1998). Animals were perfused using 4\% paraformaldehyde in PBS. Brains were post-fixed in the same fixative overnight at $4^{\circ} \mathrm{C}$ and cryoprotected overnight at $4^{\circ} \mathrm{C}$ in PBS containing $30 \%$ sucrose. Ten micrometer parasagittal sections were cut using a cryostat and incubated overnight at $4^{\circ} \mathrm{C}$ using CM1 antibody (dilution 1:1000) according to a protocol previously described (Namura et al., 1998). Immunocomplexes were then revealed using an anti-rabbit biotinylated antibody and DABcoupled ABC kit (Vectastain Elite ABC kit; Vector Laboratories, Burlingame, CA). In each experiment, control sections were included in which either the primary or the secondary antibody was omitted. Adjacent sections stained with cresyl violet were used to quantify the total number of Purkinje cells per section. To test for the presence of immunolabeled cells in the inferior olive, $14 \mu \mathrm{m}$ coronal sections of the brainstem were immunostained using the same protocol. Given the small number of positive cells, counts were performed on at least 15 sections per each animal, and results were expressed as the number of positive cells per 10 sections.

Electron microscopy. The animals were anesthetized with an intraperi- toneal injection of $3.5 \%$ chloralhydrate $(0.1 \mathrm{ml} / 10 \mathrm{gm}$ weight $)$ and perfused with $0.1 \mathrm{M}$ phosphate buffer, $\mathrm{pH} 7.4$, containing $4 \%$ paraformaldehyde and $0.1 \%$ glutaraldehyde. Brains were post-fixed overnight in $4 \%$ paraformaldehyde and processed for pre-embedding caspase- 3 immunohistochemistry using biotinylated secondary antibody and DAB-coupled $\mathrm{ABC}$ revelation kit (Vectastain Elite $\mathrm{ABC}$ kit). One hundredmicrometer-thick sections were post-fixed using $1 \%$ osmic acid in $0.1 \mathrm{M}$ PBS, pH 7.4, and embedded in Poly/Bed 812 resin (Polysciences, Warrington, PA). Ultrathin sections were cut with an ultramicrotome and stained using Reynold's lead.

To perform post-embedding immunoelectron microscopy, cerebellar slices were cut from brains fixed as for the pre-embedding experiments. These sections were dehydrated and embedded in LR white resin (London Resin Company Ltd., Reading, UK) at $50^{\circ} \mathrm{C}$. Ultrathin sections were harvested on nickel grids. These grids were used for immunohistochemistry with anti-hamster caspase-3 antibody. Immunolabeling was detected using a $10 \mathrm{~nm}$ gold-coupled goat anti-rabbit antibody (British Biocell International, Cardiff, UK). Grids were then stained for $10 \mathrm{~min}$ in $4 \%$ uranyl acetate and 1 min in Reynold's lead.

DNA fragmentation detection and double-labeling with CM1 antibody. DNA fragmentation was detected in situ using two different protocols. First, we used a one-step protocol, the Apoalert DNA fragmentation assay kit (Clontech, Palo Alto, CA), based on the terminal deoxynucleotidyl transferase-mediated dUTP nick end labeling (TUNEL) method. This assay allows the direct labeling of the fragmented DNA with fluorescein. Tissue sections from the animals used for immunohistochemistry with the anti-hamster caspase-3 antibody were processed with the Apoalert DNA fragmentation assay kit. Negative controls consisted of tissue sections in which the terminal deoxynucleotidyl transferase was deleted from the reaction mixture. The second protocol consisted of a two-step in situ DNA fragmentation assay. Briefly, brains were fixed using $4 \%$ paraformaldehyde in $0.1 \mathrm{M}$ phosphate buffer, $\mathrm{pH} 7.4$, dehydrated and paraffin-embedded. Ten micrometer parasagittal sections were rehydrated and treated with proteinase $\mathrm{K}(1 \mu \mathrm{g} / \mathrm{ml})$ for $10 \mathrm{~min}$ at room temperature. The DNA labeling reaction was performed at $37^{\circ} \mathrm{C}$ for $1 \mathrm{hr}$ using $0.3 \mathrm{U} / \mu \mathrm{l}$ terminal transferase (Boehringer Mannheim, Meylan, France) and $20 \mu \mathrm{M}$ biotin-16-2'-deoxyuridine-5'triphosphate (Boehringer Mannheim). Terminal transferase was omitted in the reaction mixture used for control sections. Labeling was then detected using an avidin-biotin-peroxidase complex (Vectastain Elite ABC kit, Vector Laboratories) and DAB revelation (DAB Sigmafast; Sigma).

Double-labeling experiments were performed on $10 \mu \mathrm{m}$ parasagittal cryostat sections of cerebella (obtained as for CM1 immunohistochemistry, see above). Sections were incubated overnight at $4^{\circ} \mathrm{C}$ with CM1 antibody (dilution 1:1000), washed in PBS, and used for a two-step in situ DNA fragmentation assay. The labeling reaction was performed using biotin-16-2'-deoxyuridine-5' triphosphate as described above. TUNEL labeling was then revealed using Texas Red-labeled streptavidin (dilution 1:1500). CM1 labeling was detected using a peroxidase-conjugated antirabbit antibody (dilution 1:1000) followed by FITC-conjugated tyramide deposition (NEN Life Science, Boston, MA). Control sections were included in each experiment by omitting terminal transferase or CM1 antibody or both. Results were analyzed using a Leica (Nussloch, Germany) confocal microscope.

\section{RESULTS \\ Expression of caspase-3}

To examine if there is a difference in the expression of caspase- 3 in the brains of Lurcher $(L c /+)$ versus wild-type $(+/+)$ mice, immunoblotting experiments were performed using protein extracts from hippocampus, cortex, and cerebellum of P20 mice. In $L c /+$ mice, there is large-scale Purkinje cell degeneration at P20, because most of the $L c /+$ Purkinje cell death occurs between P10 and P30 (Caddy and Biscoe, 1979). Two different antibodies raised against two distinct domains of caspase- 3 were used to analyze caspase-3 expression.

A polyclonal antibody (a kind gift of Drs. Goldstein and Brown) raised against amino acids $12-20$ of hamster caspase-3 was used (Fig. 1A). In $+/+$ extracts, two different bands were revealed using this antibody, corresponding to two different forms of caspase-3: pro-caspase-3 (32 kDa precursor) and the p20 sub- 
A

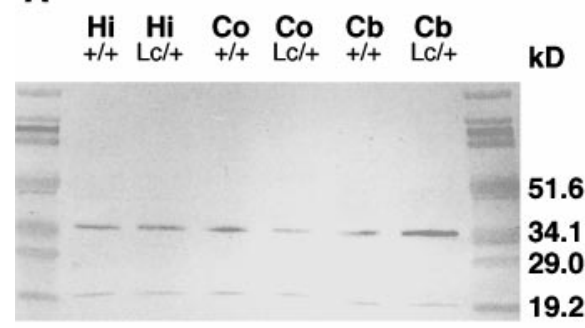

B

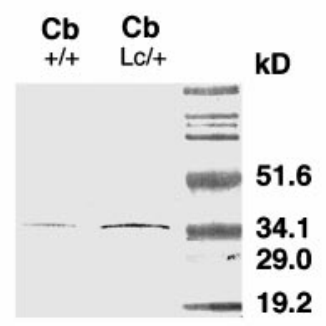

C

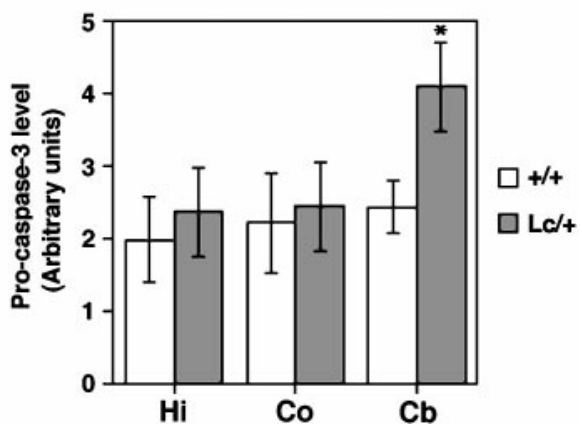

Figure 1. Caspase-3 expression in the brain of Lurcher and wild-type P20 mice. Protein extracts from hippocampus $(\mathrm{Hi})$, cortex $(\mathrm{Co})$, and cerebellum $(\mathrm{Cb})$ of Lurcher $(\mathrm{Lc} /+)$ and wild-type $(+/+)$ mice were analyzed by immunoblotting using two antibodies recognizing distinct regions of caspase-3. A, Anti-hamster caspase-3 antiserum. B, R280 antibody that specifically recognizes pro-caspase-3. $C$, Pro-caspase-3 levels revealed by the anti-hamster caspase-3 antiserum $\left(n=3 ;{ }^{*} p<0.05\right.$; Student's $t$ test).

unit of the active caspase-3, as described by Wang et al. (1996). In $L c /+$, both caspase-3 forms were also detected by immunoblotting. An increased expression of pro-caspase- 3 was consistently observed in the extracts of $L c /+$ cerebella compared with the $+/+$ extracts (Fig. 1A,C) The quantity of p20 subunit present in the cerebellum did not change in $L c /+$ mice. No difference in the quantities of pro-caspase- 3 and p20 subunit was detected in the hippocampus and cortex of $L c /+$ compared with $+/+$ mice.

Using the same protein extracts, immunoblottings were performed with another antibody raised against the large subunit of caspase-3. This antibody, named R280 (a kind gift of Dr. Nicholson), recognizes exclusively the precursor form of this protease (Mancini et al., 1998). Pro-caspase-3 was again detected in the protein extracts from cortex, hippocampus, and cerebellum of P20 $+/+$ mice. The molecular weight of the protein revealed by R280 antibody corresponded exactly to the highest molecular weight protein revealed by the anti-hamster caspase- 3 antibody, confirming that both antibodies recognized the same protein. In $L c /+$ cerebellum extracts, a clear increase in the level of pro-caspase-3 was again detected when compared to $+/+$ extracts (Fig. 1B),
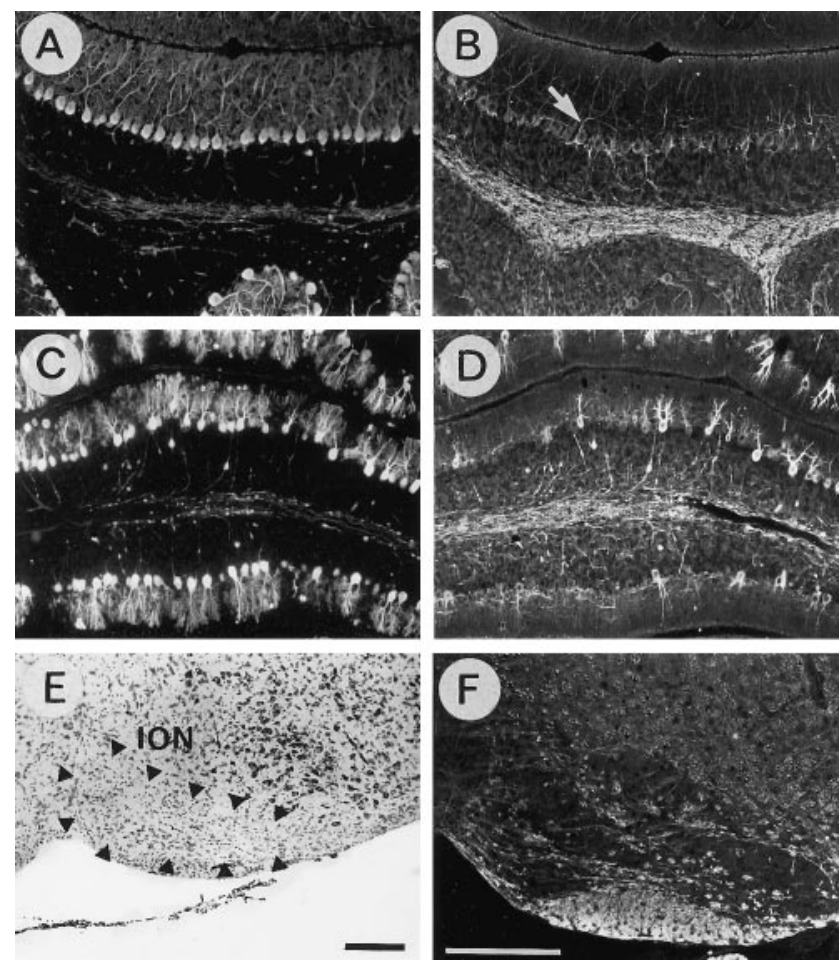

Figure 2. Immunohistochemical localization of pro-caspase-3 in the brains of P15 Lurcher and wild-type mice. Anti-calbindin $(A, C)$ and anti-caspase-3 $(B, D)$ double-immunostaining was performed on parasagittal sections of wild-type $(A, B)$ and Lurcher $(C, D)$ mice brains. Coronal sections of the Lurcher brainstem containing the inferior olive (arrowheads) were stained with cresyl violet $(E)$ and anti-caspase-3 antiserum $(F)$. Scale bars: $A-D, F, 200 \mu \mathrm{m} ; E, 200 \mu \mathrm{m}$.

whereas no change was observed in $L c /+$ cortex and hippocampus (data not shown).

These results demonstrate that pro-caspase-3 expression is specifically increased in the cerebellum of $L c /+$ mice.

\section{Localization of caspase-3 in wild-type mice}

Immunohistochemistry was performed using the anti-hamster caspase-3 antibody to localize caspase- 3 in the brain of $+/+$ mice. In $+/+$ mice, at all ages examined (P12, P15, and P20), caspase-3 immunostaining was mainly restricted to fiber-like processes (Fig. $2 B$ ). Caspase-3 immunostaining of fibers is clearly illustrated in the white matter of the cerebellum. These fibers were labeled from the white matter, through the granule cell layer to the Purkinje cell layer, and were occasionally observed penetrating the molecular layer (Fig. 2B). Some of these caspase-3immunopositive fibers were double-labeled by anti-calbindin antibodies (Fig. $2 A$ ), indicating that these fibers were Purkinje cell axons. However, not all of the caspase-3-immunopositive fibers were labeled by anti-calbindin, indicating that other categories of fibers in the cerebellum contained caspase-3. Many of the calbindin-immunonegative fibers enveloped the soma of Purkinje cells and in some instances climbed the dendritic tree of the Purkinje cell (Fig. 2B). This morphology is characteristic of the axons of inferior olivary neurons, the cerebellar climbing fibers, indicating that this category of axons also contains caspase-3.

A polyclonal antibody directed against the $\mathrm{p} 20$ subunit of active caspase-3 (CM1 antibody, a kind gift of Dr. Srinivasan) was used for immunohistochemistry on frozen brain sections of P15 and P20 +/+ mice to analyze the localization of active caspase-3. In 
Table 1. Mean number of labeled granule cells per section in P15 +/+ and $L c /+$ cerebella

\begin{tabular}{lcl} 
& Active-caspase-3 & TUNEL \\
\hline$+/+$ & $7.4 \pm 1.1$ & $14.2 \pm 1.1$ \\
$L c /+$ & $69.6 \pm 17.6^{a}$ & $90.2 \pm 23.0^{a}$
\end{tabular}

Results are expressed as mean \pm SEM; $n=3$ animals.

${ }^{a} p<0.05$ between $+/+$ and $L c /+$; ANOVA; post hoc analysis: Newman-Keuls.

$+/+$ cerebellum, Purkinje cells were consistently immunonegative, and very few granule cells were labeled (see Fig. 4A, Table 1 for quantification). The presence of few immunopositive granule cells is consistent with the fact that developmentally regulated cell death occurs in the granule cell population during postnatal development. Occasionally, labeled neurons were also found in the inferior olive of $\mathrm{P} 15+/+$ animals $(3.75 \pm 1.25$ labeled neurons per 10 sections). No immunostaining was found in the white matter of $+/+$ cerebellum, neither in the neurons of the hippocampus or the cortex of $+/+$ mice.

A comparison of the immunohistochemistry results using both antibodies indicates that active caspase- 3 is present only in a few granule cells of the $+/+$ cerebellum and a few neurons of the $+/+$ inferior olive. Anti-hamster caspase-3 immunostaining was localized to fiber-like processes and not present in any granule cell, showing that this immunostaining in fiber-like processes is specific to pro-caspase-3.

\section{Localization of caspase-3 in Lurcher mice}

To identify which category of cerebellar cells overexpresses procaspase-3 in $L c /+$ mice, anti-hamster caspase-3 immunostaining was performed on brain sections of mutant mice.

In the cerebellum of $L c /+$ mice at all ages examined (P12, P15, and P20), caspase-3 immunostaining was strikingly intense in some, but not all, Purkinje cells (Fig. 2D). Caspase-3 immunostaining was not detected in the cell body of any other cell type in the cerebellum (Fig. 2D). In the cerebellar nodulus, where the Purkinje cells are the last to degenerate in the $L c /+$ mice, no caspase-3-immunopositive Purkinje cell bodies and dendrites were observed at any of the three ages examined. In coronal sections of the cerebellum and the brainstem, no staining was observed in the inferior olivary neurons (Fig. $2 F$ ). These observations demonstrate that not all the categories of cells that die in the $L c /+$ brain are caspase-3-immunopositive. The caspase-3 immunoreactivity is specific to dying $L c /+$ Purkinje cells. In other regions of the brain not affected by the $L c /+$ mutation, no neuron was found to be immunopositive for caspase-3. For example, in the hippocampus where no difference of expression of caspase-3 was detected by immunoblotting, no cell body was immunostained. These observations in $L c /+$ mice indicate a correlation between the caspase- 3 immunoreactivity of Purkinje cells, the increase of pro-caspase-3 observed in the cerebellum by immunoblotting and Purkinje cell death. Caspase- 3 immunostaining was also present in fiber-like processes throughout the brain of $L c /+$ animals, consistent with the result obtained from brain sections of $+/+$ mice.

Interestingly, the localization of pro-caspase-3 was not the same in every immunopositive Purkinje cell. At all ages examined, numerous Purkinje cells were strongly immunoreactive in the perinuclear region of the soma and the dendritic shafts. Some Purkinje cells were stained only in the perinuclear soma, others were also stained in the major dendrites and, sometimes,
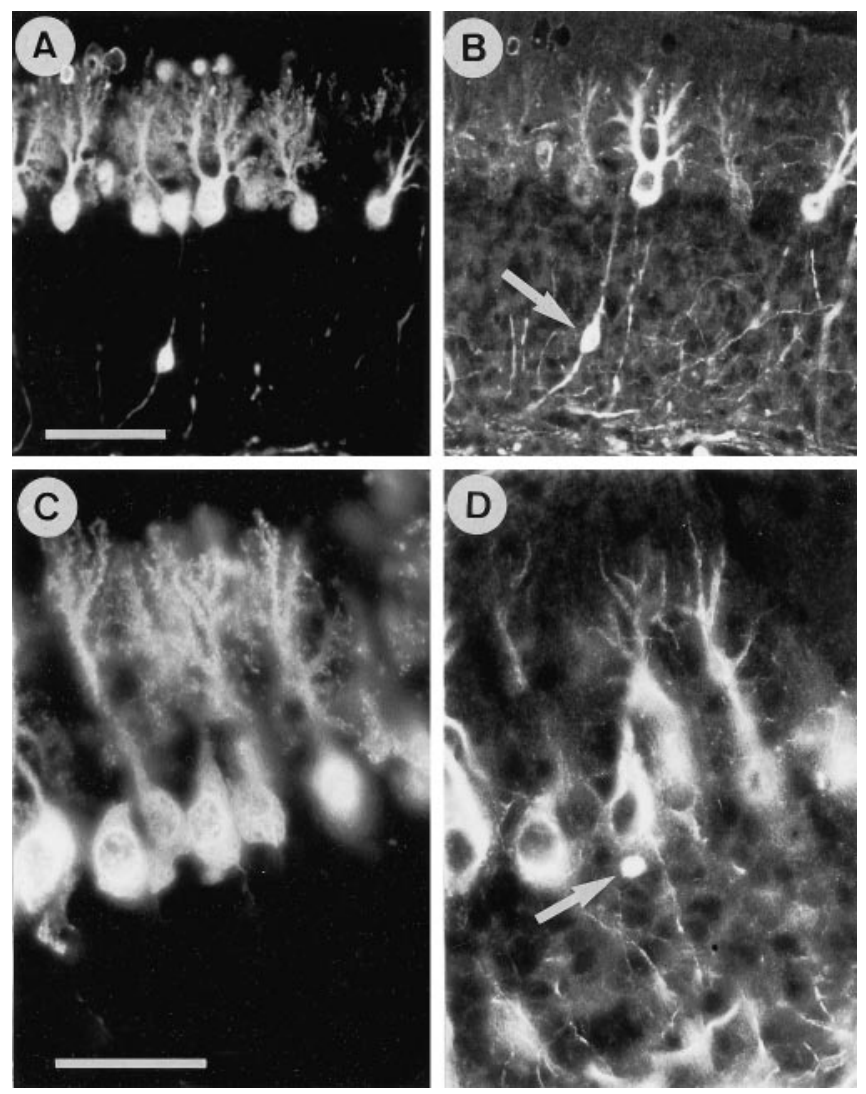

Figure 3. Pro-caspase-3 localization in Lurcher Purkinje cells. Procaspase-3 localization in axonal varicosities is shown at a higher magnification in P15 Lurcher Purkinje cells ( $B$, arrow). Note that the calbindinimmunostained cell body $(A)$ is not labeled by the pro-caspase-3 antiserum. $D$ shows the more confined localization of pro-caspase- 3 in some Purkinje cell somas as well as the fact that the dendritic spines, visible with the calbindin immunostaining $(C)$, are not pro-caspase-3immunoreactive. Note also the immunostained varicosity (arrow) that could belong to a recurrent collateral axon of a Purkinje cell. Scale bars: $A, B, 40 \mu \mathrm{m} ; C, D, 20 \mu \mathrm{m}$.

caspase-3 staining could be observed within smaller dendrites, thus extending to the upper part of the dendritic arborization. Pro-caspase-3 was not present in the terminal parts of the dendrites, because the immunostaining did not reveal the spiny branchlets that can be seen with anti-calbindin immunostaining (Fig. 3). The axons of degenerating Purkinje cells in $L c /+$ mice are characterized by the presence of varicosities. These axonal varicosities were intensely stained by caspase- 3 immunohistochemistry. In particular, these axonal varicosities could be strongly caspase-3 immunopositive and the soma of the same Purkinje cell immunonegative (Fig. $3 A, B$ ). In sections of $\mathrm{P} 12$ $L c /+$ mice, some Purkinje cells were stained in a more confined region of their soma (Fig. 3D). This observation suggests a confined localization of pro-caspase- 3 at the beginning of the cell death process. In addition, some fibers that terminated in a varicosity on the Purkinje cell soma were intensely stained by caspase-3 antibody in sections of P12 Lc/ + mice (Fig. $3 D$ ). The morphology of these fibers is similar to that of the recurrent collateral axons of Purkinje cells. These fibers were calbindinimmunoreactive, supporting a Purkinje cell origin.

As we have previously noted, not all the Purkinje cells in $\mathrm{Lc} /+$ cerebellum were immunopositive. The percentage of procaspase-3 positive Purkinje cells was roughly the same at each age 
Figure 4. Active caspase-3 and TUNEL staining in Lurcher cerebellum. Immunostaining was performed on parasagittal sections of P15 $(A-C)$ and P20 $(D)$ cerebella using an antibody that specifically recognizes the p20 subunit of active caspase-3. No cells are immunostained in wild-type cerebellum $(A)$, whereas active caspase-3 can be found in Purkinje cells and granule cells in Lurcher cerebellum $(B-D)$. TUNEL staining was performed on parasagittal sections of P15 cerebella. Numerous granule cells are labeled in the Lurcher cerebellum $(F)$ in contrast to the staining pattern observed in the wild-type $(E)$. A high magnification in $F$ shows the labeling of a Lurcher Purkinje cell nuclei. Scale bars: $A-C, E, F, 100 \mu \mathrm{m} ; D, 50 \mu \mathrm{m}$; high magnification in $F$, $20 \mu \mathrm{m}$.
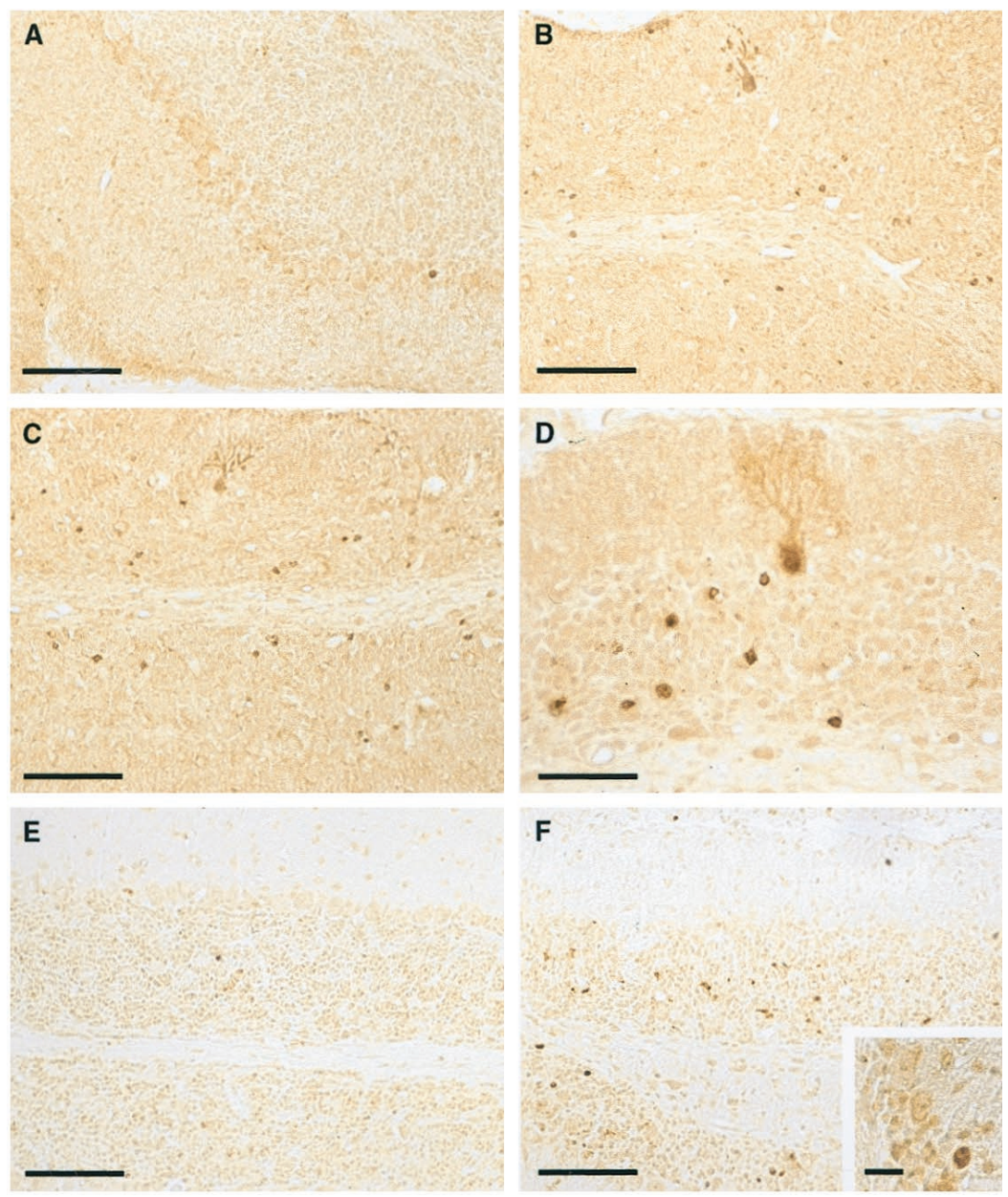

examined (20-25\%), suggesting that the overexpression of procaspase-3 in $L c /+$ Purkinje cells is not developmentally regulated but is rather related to the degenerative state of the Purkinje cell.

To test whether caspase- 3 was active in the dying $L c /+$ cells, immunohistochemistry using CM1 antibody recognizing the p20 subunit of active caspase-3 was performed on brain sections of $L c /+$ mice (P15 and P20). Strongly immunoreactive granule cells and Purkinje cells were found in $L c /+$ cerebellum (Fig. $4 B-D$ ). Fibers in the white matter of the cerebellum were immunonegative. No immunolabeled neurons could be found in the hippocampus and the cortex of $L c /+$ mice (which are not a priori affected by the $L c$ mutation). The percentage of immunopositive Purkinje cells (between 1 and 3\%) was very low compared to the percentage of cells immunopositive for the caspase-3 precursor, showing that the delay between caspase- 3 activation and Purkinje cell death is very short in comparison with the period of procaspase-3 overexpression. The number of labeled granule cells per section was considerably increased in $L c /+$ cerebellum compared to the $+/+$. This number was quantified in P15 animals, and the increase was found to be statistically significant (Table 1). Cell counts in the inferior olive of $\mathrm{P} 15 \mathrm{Lc} / \mathrm{+}$ mice reveal $13.86 \pm$ 0.02 labeled neurons per 10 sections. This number is higher than the number of labeled neurons in P15 $+/+$ inferior olive and corresponds to a sixfold increase in the percentage of labeled olivary neurons, when the $40 \%$ loss of $L c /+$ inferior olivary neurons at P15 is taken into account (Caddy and Biscoe, 1979). The presence of active caspase- 3 in granule cells and Purkinje cells of $L c /+$ cerebella was confirmed by immunohistochemical experiments using another antibody directed against active caspase-3 (MF397, a kind gift of Dr. Nicholson; data not shown). The fact that active caspase- 3 could be found in granule cells and olivary neurons, whereas no pro-caspase- 3 was detected in these cells, could be explained by a different sensitivity of the two antibodies used. For instance, the anti-hamster caspase-3 might only immunocytochemically label high levels of pro-caspase-3 in cells, such as observed in $L c /+$ Purkinje cells.

These results demonstrate that pro-caspase- 3 is upregulated in dying $L c /+$ Purkinje cells. Active caspase- 3 is also found in $L c /+$ Purkinje cells. In addition, active caspase- 3 is present in granule cells and inferior olivary neurons that die extensively in the $L c /+$ cerebellum because of the loss of their target.

\section{Ultrastructural localization of pro-caspase-3}

To assess the ultrastructural localization of pro-caspase-3 in $\mathrm{Lc} /+$ Purkinje cells, we performed immunoelectron microscopy using the anti-hamster caspase-3 antibody on cerebellar sections of P15 $+/+$ and $L c /+$ mice.

When examined under the electron microscope, immunolabeled $L c /+$ cerebellar sections exhibited morphological abnormalities associated with the mutation such as Purkinje cell loss and reduced molecular layer. Chromatin clumps and swollen mitochondria with dilated cristae were observed in $L c /+$ Purkinje cells, as previously described (Dumesnil-Bousez and Sotelo, 1992). Pro-caspase-3 immunostaining was present in the soma and dendrites of degenerating Lc/+ Purkinje cells (Fig. 5B,C). The immunoprecipitates were cytoplasmic, but also localized in 

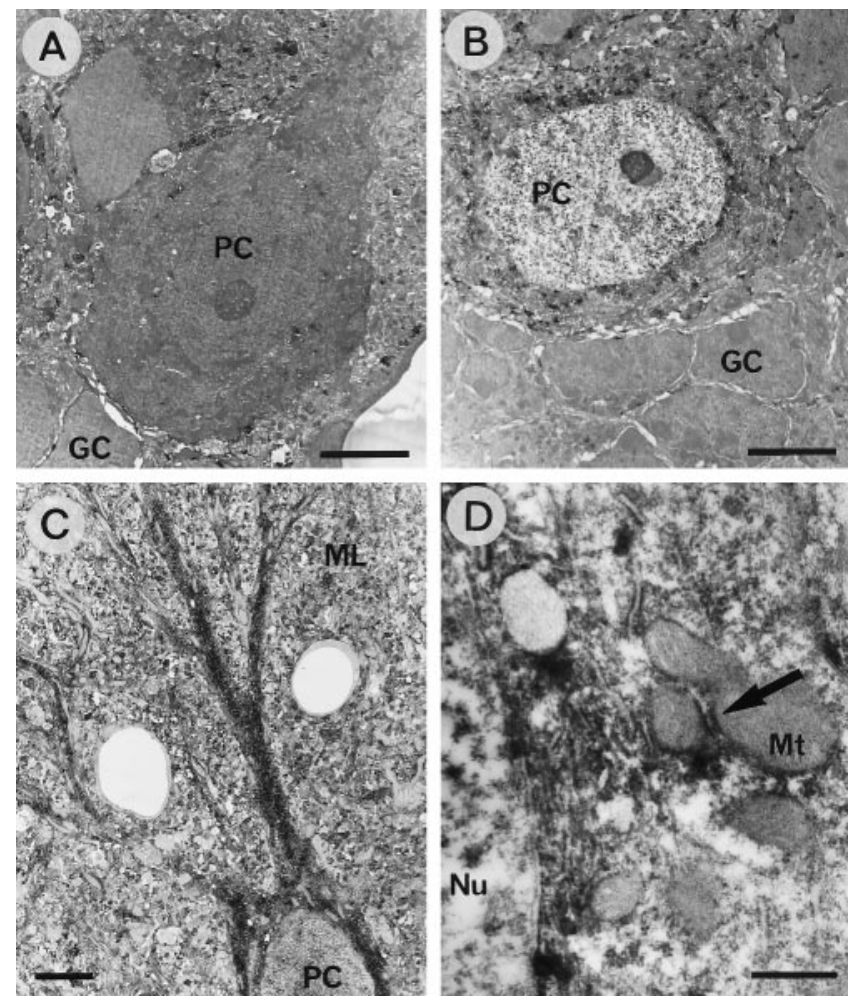

Figure 5. Ultrastructural localization of pro-caspase-3. Post-embedding immunoelectron microscopy was performed on cerebellar sections of P15 wild-type and Lurcher mice. Wild-type Purkinje cells and granule cells are not stained $(A)$, whereas pro-caspase-3 immunostaining can be observed in Purkinje cells and in fibers located in the molecular layer of Lurcher cerebellum $(B-D)$. Mitochondrial staining is shown at a high magnification ( $D$, arrow). $P C$, Purkinje cell; $G C$, granule cell; $M L$, molecular layer; $M t$, mitochondrion; $N u$, nucleus. Scale bars: $A-C, 5 \mu \mathrm{m} ; D$, $0.5 \mu \mathrm{m}$.

the outer membrane of some mitochondria (Fig. 5D). To verify that the mitochondrial staining was not an experimental artifact, we performed postembedding immunostaining using a goldcoupled secondary antibody on ultrathin cerebellar sections of P15 mice cerebella. Gold particles were observed in the mitochondria of Purkinje cells, as well as in their cytoplasm (data not shown), showing that a fraction of pro-caspase-3 is actually present in the mitochondria of $L c /+$ Purkinje cells. Purkinje cells in $+/+$ cerebellar sections did not display any immunostaining in their soma and dendrites (Fig. 5A). Immunoprecipitates could be found in fibers localized in the white matter, the granule cell layer, and the molecular layer of $+/+$ cerebella in accordance with what was observed under the optic microscope.

These results demonstrate that pro-caspase- 3 is localized in the cytoplasm and the mitochondria of dying $L c /+$ Purkinje cells.

\section{Comparison between DNA fragmentation and caspase- 3 activation}

Fragmentation of nuclear DNA is considered to be a general feature of cells undergoing apoptosis. To test if $L c /+$ Purkinje cells expressing caspase-3 also exhibit this characteristic, we performed an assay based on the TUNEL method on brain sections of P15 Lc/ + and $+/+$ mice.

Using a one-step method, DNA fragmentation was detected in numerous granule cells in the cerebellum of $L c /+$ mice at a much higher frequency when compared to $+/+$, but no Purkinje cells were labeled using this DNA fragmentation assay in the sections analyzed (data not shown).

To increase the sensitivity of detection, we then used a two-step in situ DNA fragmentation labeling method. Numerous granule cells were again labeled in the internal granule layer of the $L c /+$ cerebellum (Fig. $4 F$ ). The number of labeled $L c /+$ granule cells was significantly higher than the number of granule cells labeled in the $+/+$ cerebellum (Fig. $4 E, F$; see Table 1 for quantification), consistent with the fact that numerous granule cells die in the $L c /+$ cerebellum after the loss of their target, the Purkinje cell. Rarely, some Purkinje cell nuclei were labeled in $L c /+$ cerebella using this two-step technique as shown in Figure $4 F$, whereas Purkinje cell nuclei were never labeled in $+/+$ cerebella. We estimated the number of labeled $L c /+$ Purkinje cells to be $<0.5 \%$. This result suggests that DNA fragmentation in the $L c /+$ Purkinje cell is quickly followed by phagocytosis and thus very difficult to detect.

We found that the number of TUNEL-positive granule cells and the number of active caspase-3-positive granule cells were not statistically different in $L c /+$ cerebellum (Table 1 ). This suggests that the time courses of caspase- 3 activation and DNA fragmentation in Lc/ + granule cells are similar. These events are easier to detect in granule cells than in Purkinje cells, because the total number of granule cells per section is very high.

Double-labeling experiments on $L c /+$ cerebellar sections were performed using anti active caspase-3 antibody (CM1 antibody) and a two-step in situ DNA fragmentation assay. Numerous granule cells were found to be double-labeled as shown in Figure 6, confirming that granule cell death in $L c /+$ cerebellum is an apoptotic process involving caspase- 3 activation and DNA fragmentation. Not all caspase-3-labeled granule cells were TUNELpositive and vice versa. This indicates that these two molecular events are only partially overlapping during neuronal death, as already suggested by Namura et al. (1998). These experiments did not reveal double-labeled Purkinje cells, despite the fact that we screened sections from several animals. The number of either active caspase-3-immunopositive or TUNEL-positive Purkinje cells are low in $L c /+$ cerebella. Considering evidence from granule cells suggests that these two events are only partially overlapping, the probability of detecting double-labeled cells in the much smaller Purkinje cell population is remote.

\section{DISCUSSION}

We have shown that caspase-3 is upregulated and activated in dying Lurcher $(L c /+)$ Purkinje cells. Pro-caspase-3 was detected in the perinuclear soma as well as in the dendritic shafts of Purkinje cells. Its subcellular localization was shown to be cytoplasmic and partly mitochondrial. Active caspase-3 was detected in dying granule cells and olivary neurons in the $L c /+$ brain, but we did not observe any upregulation of pro-caspase- 3 in these cell types. DNA fragmentation was found using the TUNEL technique in the nuclei of dying granule cells and a few Purkinje cells in the cerebellum of $L c /+$ mice. These observations suggest that the death of Purkinje cells and granule cells in $L c /+$ mice is apoptotic and mediated by caspase-3 activity.

\section{Caspase- 3 is constitutively expressed in $+/+$ brain}

A constitutive expression of caspase- 3 was revealed by immunoblotting in the cortex, hippocampus, and cerebellum of $+/+$ mice in agreement with the results obtained by other authors (Krajewska et al., 1997; Chen et al., 1998; Namura et al., 1998). We demonstrated that pro-caspase-3 is targeted to fiber-like pro- 

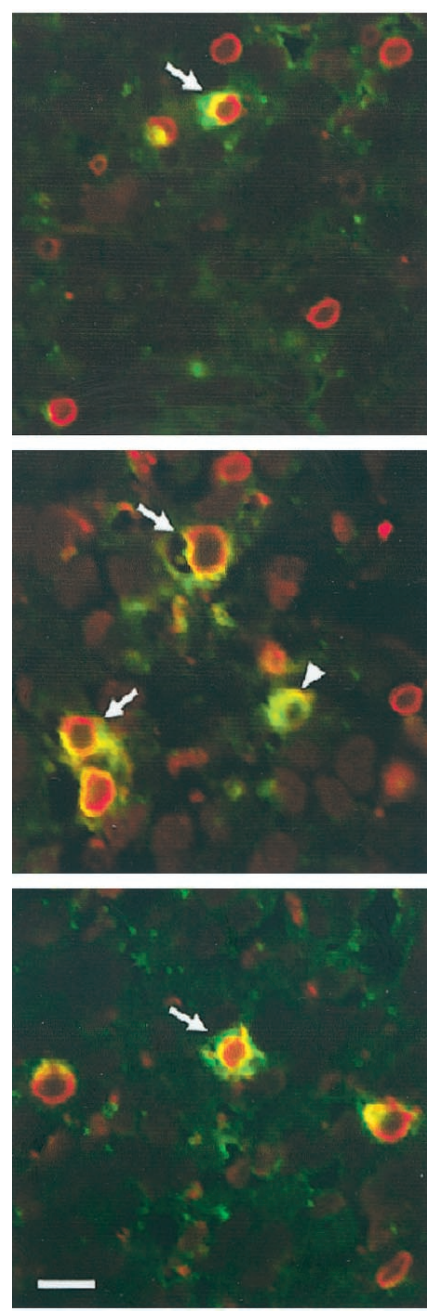

Figure 6. Active caspase-3 and DNA fragmentation are simultaneously detected in some granule cells of Lurcher cerebellum. A number of granule cells are stained by CM1 antibody (green, arrowhead), another subset of granule cells are only TUNEL-positive (red), and some are double-labeled (arrow). Micrographs were obtained using confocal microscope. Scale bar, $10 \mu \mathrm{m}$.

cesses in the $+/+$ brain, and we could not detect immunolabeled nerve cell bodies in agreement with Chen et al. (1998) and Krajewska et al. (1997). Namura et al. (1998) could detect procaspase-3 in fiber-like processes and in some neuronal somas. These observations suggest that if pro-caspase- 3 is present in neuronal somas, its level is quite low and not easily detectable by immunohistochemistry. In our preparation, some cerebellar granule cells of the $+/+$ mice contained active caspase- 3 , which is likely to be caused by developmentally regulated cell death (Herrup and Busser, 1995). Taken together, these results indicate that some categories of neurons express the apoptotic machinery even in the normal postnatal brain.

\section{Target-related cell death versus intrinsic neuronal death in Lc/+ cerebellum}

Studies on Lurcher chimeric mice have shown that the cerebellum of $L c /+$ mice is affected by two types of neurodegeneration. The degeneration of Purkinje cells is a direct consequence of the presence of a mutated GluR $\delta 2$ protein, whereas the death of olivary neurons and granule cells is an indirect effect and presumably follows the loss of their target neuron, the Purkinje cell
(Herrup et al., 1996; Wetts and Herrup, 1982b). Comparing both types of neuronal death in $L c /+$ mice suggests that different pathways are triggered depending on which death signal the neuron receives. Two phases can be distinguished during the death process taking place in $L c /+$ Purkinje cells: the first phase involves an increase in the expression of pro-caspase-3, followed by a subsequent triggering of caspase- 3 activation engaging the Purkinje cell in the last phase of its destruction. Zanjani et al. (1998a) demonstrated that the overexpression of the antiapoptotic protein $\mathrm{Bcl}-2$ does not rescue the $L c /+$ Purkinje cells. Thus, the intrinsic neuronal death affecting $L c /+$ Purkinje cells is Bcl-2 independent and mediated by the upregulation and activation of caspase-3. In parallel, pro-caspase-3 is not upregulated during the target-related death of the granule cells and olivary neurons in $L c /+$ mice. However, numerous granule cells contain the active caspase- 3 and are TUNEL-positive in $L c /+$ cerebellum. Some are double-labeled confirming the apoptotic nature of granule cell death. Target-related cell death of granule cells has already been associated with TUNEL staining in the Lurcher cerebellum (Wüllner et al., 1995) and also in a model where Purkinje cells were ablated by the specific expression of diphtheria toxin (Smeyne et al., 1995). The presence of active caspase-3 is also detected in some olivary neurons in $L c /+$ brainstem. In contrast to Purkinje cells, the overexpression of Bcl-2 in Lurcher does rescue inferior olivary neurons (Zanjani et al., 1998a). Bcl-2 was not overexpressed in the granule cells in this transgenic model of Bcl-2 overexpression. However, granule cells from these mice overexpress $\mathrm{Bcl}-2$ in primary cultures and are resistant to various death stimuli (Tanabe et al., 1997). Thus, on the basis that granule cells and inferior olivary neurons are subjected to similar cell death stimuli, we can speculate that granule cells would also be rescued by Bcl-2 overexpression in vivo. Taken together, these observations suggest that the target-related cell death of granule cells and olivary neurons in $L c /+$ mice involves a variant apoptotic pathway that is $\mathrm{Bcl}-2$ dependent and does not require procaspase-3 upregulation but is mediated by caspase- 3 activation. Despite these differences, our results indicate a central role of caspase-3 in both types of neuronal death observed in $L c /+$ mice. The nature of the Purkinje cell death in $L c /+$ cerebellum is controversial, because morphological observations have suggested a necrotic death (Dumesnil-Bousez and Sotelo, 1992) and other studies an apoptotic mechanism (Norman et al., 1995; Wüllner et al., 1995, 1998). Caspases have been shown to be key executioners of apoptosis (Green and Kroemer, 1998; Thornberry and Lazebnik, 1998) and therefore our results strongly support the idea that $L c /+$ Purkinje cell death is apoptotic.

\section{Apoptosis in Lc/+ Purkinje cell}

The genetic defect responsible for Purkinje cell degeneration in $L c /+$ mice is a point mutation in the gene encoding the GluR $\delta 2$ protein. This protein has an unknown function, but the $L c$ mutation in this protein leads to the presence of a constitutive inward current in the $L c /+$ Purkinje cells that maintains these cells at a depolarized resting membrane potential. These observations have led to the idea that the $L c /+$ Purkinje cell death is induced by excitotoxicity (Zuo et al., 1997). However, the molecular mechanism underlying this neuronal death has not been resolved. We suggest that the $L c /+$ Purkinje cell death might involve two phases. During the first phase, an upregulation of caspase-3 expression is induced. It is interesting to note that such an upregulation has already been revealed in another model of excitotoxicity, ischemia (Chen et al., 1998; Namura et al., 1998). 
In addition, caspase- 3 overexpression is able to induce apoptosis in vitro (Fernandes-Alnemri et al., 1994). These results suggest that the upregulation of pro-caspases is also involved in the induction of neuronal apoptosis in vivo, especially in the case of a chronic exposure to a death signal such as occurs in $L c /+$ Purkinje cells. Although Bcl-2 overexpression does not inhibit $L c /+$ Purkinje cell death, it is able to delay the death process, as, unlike in control $L c /+$ mice, Purkinje cells can be found in 2-month-old $L c /+$ mice overexpressing Bcl-2 (Zanjani et al., 1998b). Interestingly, Bcl-2 has been shown to downregulate caspase-3 expression in neuronal precursors (Korhonen et al., 1997). Therefore, Bcl-2 might downregulate temporarily the overexpression of caspase-3 in $L c /+$ Purkinje cells and might also inhibit the effect of Bax, a pro-apoptotic protein, which has recently been shown to be upregulated in dying $L c /+$ Purkinje cells (Wullner et al., 1998). But, as the abnormal inward current induced by the Lurcher mutation of GluR $\delta 2$ in $L c /+$ Purkinje cells is continually present (Zuo et al., 1997), Bcl-2 overexpression might become insufficient.

Mancini et al. (1998) have shown that the fraction of procaspase-3 present in mitochondria disappears after the induction of apoptosis. Pro-caspase-3 was detected in the mitochondria of dying $L c /+$ Purkinje cells overexpressing pro-caspase-3, indicating that these cells might be in the first phase of their death process when apoptosis is not yet fully triggered. The first morphological signs of $L c /+$ Purkinje cell abnormalities can be detected as early as P6-P8 (Dumesnil-Bousez and Sotelo, 1992; Bailly et al., 1996). Interestingly, one of these signs is a swelling of the mitochondria, a feature that has been observed in some models of apoptosis (Vander Heiden et al., 1997). The death signal in $L c /+$ Purkinje cell might also induce a mitochondrial dysfunction and the subsequent release, at an adequate time, of mitochondrial components that have been shown to induce caspase- 3 activation and the subsequent destruction of the cell, e.g., cytochrome c or apoptosis inducing factor (Green and Kroemer, 1998). Finally, both pathways, mitochondrial dysfunction and caspase activation, might interact to amplify each other as it has recently been proposed by Green and Kroemer (1998), leading to a rapid final destruction of the $L c /+$ Purkinje cells. In fact, the actual destruction of the cell, the second phase of $L c /+$ Purkinje cell death, might be triggered only once a certain threshold of mitochondrial dysfunction and death protein overexpression is reached. This destruction ends by a rapid engulfment of the remnants of the cell (Thornberry and Lazebnik, 1998). Thus, the second phase of the $L c /+$ Purkinje cell death process is likely to be more difficult to observe, a hypothesis that is supported by the fact that, in the $L c /+$ cerebellum, the number of Purkinje cells containing active caspase- 3 is quite low, and the number of TUNEL-positive Purkinje cells even lower.

Our study provides evidence for the central role of caspase- 3 in two types of pathological neuronal death: a target-related cell death and an intrinsic neuronal death of genetic origin. Moreover, the chronic exposure to a death signal, such as occurs in $L c /+$ Purkinje cells, may induce a preliminary phase involving an upregulation of caspase- 3 and other death proteins, as well as mitochondrial dysfunction. In contrast, this preliminary phase is absent in the target-related death of granule cells.

\section{REFERENCES}

Araki K, Meguro H, Kushiya E, Takayama C, Inoue Y, Mishina M (1993) Selective expression of the glutamate receptor channel $\delta 2$ subunit in cerebellar Purkinje cells. Biochem Biophys Res Commun 197:1267-1276.
Bailly Y, Kyriakopoulou K, Delhaye-Bouchaud N, Mariani J, Karagogeos D (1996) Cerebellar granule cell differentiation in mutant and $\mathrm{X}$-irradiated rodents revealed by the neural adhesion molecule TAG-1. J Comp Neurol 369:150-161.

Caddy KWT, Biscoe TJ (1979) Structural and quantitative studies on the normal $\mathrm{C} 3 \mathrm{H}$ and Lurcher mutant mouse. Philos Trans R Soc Lond B Biol Sci 287:167-201.

Chen J, Nagayama T, Jin K, Stetler RA, Zhu RL, Graham SH, Simon RP (1998) Induction of caspase-3-like protease may mediate delayed neuronal death in the hippocampus after transient cerebral ischemia. J Neurosci 18:4914-4928.

Du Y, Dodel RC, Bales KR, Jemmerson R, Hamilton-Byrd E, Paul SM (1997) Involvement of a caspase-3-like cysteine protease in 1-methyl4-phenylpyridinium-mediated apoptosis of cultured cerebellar granule neurons. J Neurochem 69:1382-1388.

Dumesnil-Bousez N, Sotelo C (1992) Early development of the Lurcher cerebellum: Purkinje cell alterations and impairment of synaptogenesis. J Neurocytol 21:506-529.

Fernandes-Alnemri T, Litwack G, Alnemri ES (1994) CPP32, a novel human apoptotic protein with homology to Caenorhabditis elegans cell death protein Ced-3 and mammalian interleukin- $\beta$-converting enzyme. J Biol Chem 269:30761-30764.

Green D, Kroemer G (1998) The central executioners of apoptosis: caspases or mitochondria? Trends Cell Biol 8:267-271.

Herrup K, Busser JC (1995) The induction of multiple cell cycle events precedes target-related neuronal death. Development 121:2385-2395.

Herrup K, Shojaeian-Zanjani H, Panzini L, Sunter K, Mariani J (1996) The numerical matching of source and target populations in the CNS: the inferior olive to Purkinje cell projection. Brain Res Dev Brain Res 96:28-35.

Korhonen L, Hamnér S, Olsson P, Lindholm D (1997) Bcl-2 regulates the levels of the cysteine proteases ICH and CPP32/Yama in human neuronal precursor cells. Eur J Neurosci 9:2489-2496.

Krajewska M, Wang H, Krajewski S, Zapata JM, Shabaik A, Gascoyne R, Reed JC (1997) Immunohistochemical analysis of in vivo patterns of expression of CPP32 (caspase-3), a cell death protease. Cancer Res 57:1605-1613.

Kuida K, Zheng TS, Na S, Kuan C, Yang D, Karasuyama H, Rakic P, Flavell RA (1996) Decreased apoptosis in the brain and premature lethality in CPP32-deficient mice. Nature 384:368-372.

Mancini M, Nicholson DW, Roy S, Thornberry NA, Peterson EP, Casciola-Rosen LA, Rosen A (1998) The caspase-3 precursor has a cytosolic and mitochondrial distribution: implications for apoptotic signaling. J Cell Biol 140:1485-1495.

Marks N, Berg MJ, Guidotti A, Saito M (1998) Activation of caspase-3 and apoptosis in cerebellar granule cells. J Neurosci Res 52:334-341.

Namura S, Z hu J, Fink K, Endres M, Srinivasan A, Tomaselli KJ, Yuan J, Moskowitz MA (1998) Activation and cleavage of caspase-3 in apoptosis induced by experimental cerebral ischemia. J Neurosci 18:3659-3668.

Nicholson DW, Thornberry NA (1997) Caspases: killer proteases. Trends Biol Sci 22:299-306.

Norman DJ, Feng L, Cheng SS, Gubbay J, Chan E, Heintz N (1995) The lurcher gene induces apoptotic death in cerebellar Purkinje cells. Development 121:1183-1193.

Rodriguez I, Matsuura K, Ody C, Nagata S, Vassalli P (1996) Systemic injection of a tripeptide inhibits the intracellular activation of CPP32like proteases in vivo and fully protects mice against Fas-mediated fulminant liver destruction and death. J Exp Med 184:2067-2072.

Smeyne RJ, Chu T, Lewin A, Bian F, S-Crisman S, Kunsch C, Lira SA, Oberdick J (1995) Local control of granule cell generation by cerebellar Purkinje cells. Mol Cell Neurosci 6:230-251.

Tanabe H, Eguchi Y, Kamada S, Martinou J, Tsujimoto Y (1997) Susceptibility of cerebellar granule neurons derived from Bcl-2-deficient and transgenic mice to cell death. Eur J Neurosci 9:848-856.

Thornberry NA, Lazebnik Y (1998) Caspases: enemies within. Science 281:1312-1316.

Thornberry NA, Rano TA, Peterson EP, Rasper DM, Timkey T, GarciaCalvo M, Houtzager VM, Nordstrom PA, Roy S, Vaillancourt JP, Chapman KT, Nicholson DW (1997) A combinatorial approach defines specificities of members of the caspase family and granzyme B. J Biol Chem 272:17907-17911. 
Vander Heiden MG, Chandel NS, Williamson EK, Schumacker PT, Thompson CB (1997) Bcl- $x_{\mathrm{L}}$ regulates the membrane potential and volume homeostasis of mitochondria. Cell 91:627-637.

Wang X, Zelenski NG, Yang J, Sakai J, Brown MS, Goldstein JL (1996) Cleavage of sterol regulatory element binding proteins (SREBPs) by CPP32 during apoptosis. EMBO J 15:1012-1020.

Wetts R, Herrup K (1982b) Interaction of granule, Purkinje and inferior olivary neurons in Lurcher chimeric mice. II. Granule cell death. Brain Res 250:358-362.

Wüllner U, Löschmann P-A, Weller M, Klockgether T (1995) Apoptotic cell death in the cerebellum of mutant weaver and lurcher mice. Neurosci Lett 200:109-112.

Wullner U, Weller M, Schulz J, Krajewski S, Reed J, Klockgether T
(1998) Bcl-2, Bax and Bcl-x expression in neuronal apoptosis: a study of mutant weaver and lurcher mice. Acta Neuropathol (Berl) 96:233-238.

Zanjani HS, Vogel MW, Martinou JC, Delhaye-Bouchaud N, Mariani J (1998a) Postnatal expression of $\mathrm{Hu}-\mathrm{Bcl}-2$ gene in Lurcher mutant mice fails to rescue Purkinje cells but protects inferior olivary neurons from target-related cell death. J Neurosci 18:319-327.

Zanjani H, Rondi-Reig L, Vogel M, Martinou J-C, Delhaye-Bouchaud N, Mariani J (1998b) Overexpression of a Hu-bcl-2 transgene in Lurcher mutant mice delays Purkinje cell death. C R Acad Sci (Paris) 321:633-640.

Zuo J, De Jager PL, Takahashi KA, Jiang W, Linden DJ, Heintz N (1997) Neurodegeneration in Lurcher mice caused by mutation in $\delta 2$ glutamate receptor gene. Nature 388:769-773. 